\title{
A Multi-Analytical Approach for the Study of the pigments Used to Decorate an Egyptian Cartonnage from Ptolemaic Period
}

\author{
Faramarz S. Gard $^{1 *}$, Patricia. B. Bozzano ${ }^{1,2}$, Diego M. Santos ${ }^{3}$, Maria B. Daizo ${ }^{3,4}$, Emilia B. Halac ${ }^{1,2}$, Maria \\ Reinoso $1,2,5$ \\ ${ }^{1}$ Comisión Nacional de Energía Atómica, Buenos Aires, Argentina \\ ${ }^{2}$ Institute Sabato-UNSAM \\ ${ }^{3}$ Universidad Pedagógica Nacional, Buenos Aires, Argentina \\ ${ }^{4}$ Universidad de Buenos Aires, Argentina \\ ${ }^{5}$ CONICET \\ * Corresponding author: fsgard01@ gmail.com
}

In the current study, fragments from an Egyptian cartonnage from Ptolemaic period (305-30 BC.), which was provided by the Museum of La Plata, were analyzed in order to identify the pigments and minerals used to decorate the cartonnage. The analytical techniques employed for the characterization of the pigments were the scanning electron microscope coupled with an energy dispersive spectrometer (SEMEDS) and a electron probe micro analyzers coupled with a wavelength dispersive spectrometer (EPMAWDS), and Raman spectroscopy.

While SEM and EPMA allowed us to perform a qualitative chemical analysis and morphological studies of the samples, by Raman spectroscopy the associated minerals of the pigments have been identified. All SEM-EDS measurements, back scattering (BS), and secondary electron (SE) imaging were performed in a FEI Quanta 200 SEM with an EDAX Apollo detector, at low vacuum mode. EPMA analysis was carried out in a CAMECA SXFiveFE. Raman microscopy analyses were performed on a LabRAM HR Raman system (Horiba Jobin Yvon), equipped with two monochromator gratings and a charge coupled device detector (CCD).

Figure 1 shows a typical fragment of the cartonnage. This, among other smaller pieces, were used to analyze the pigments. The cartonnage is made of woven layers of linen, which is covered by plaster. A multicolored pattern was then painted on the plaster. The fragment, shown in Fig. $1 \mathrm{~A}$, contains rows of multicolored triangles, and a rather dark orange stripe, which is separating the rows of the triangle patterns. Golden areas in the fragment are associated to the funerary mask, which was decorated with golden leaf. A light green color region is also seen on the right hand side of the fragment which was part of the crown of the mask.

Raman spectroscopy confirmed the presence of cinnabar $(\alpha-\mathrm{HgS})$ in the red part of the sample. Whereas, a mixture of orpiment $\left(\mathrm{As}_{2} \mathrm{~S}_{3}\right)$ and bonazziite $\left(\beta-\mathrm{As}_{4} \mathrm{~S}_{4}\right)$ was detected in the yellow regions of the fragments. In the orange region a mixture of orpiment and pararealgar $\left(\mathrm{As}_{4} \mathrm{~S}_{4}\right)$, was detected. However, we have confirmed that the original orange pigment was a blend of orpiment and realgar $(\alpha-$ $\left.\mathrm{As}_{4} \mathrm{~S}_{4}\right)$, that has transformed to pararealgar due to the exposure to light. Egyptian blue $\left(\mathrm{CaCuSi}_{4} \mathrm{O}_{10}\right)$ and Egyptian green $(\mathrm{Cu}, \mathrm{Ca}) \mathrm{SiO}_{3}$ pigments were also detected from blue/green dark colored regions of the fragments. Figure 2 panels (A, B) show characteristic Backscattering (BS) images obtained from the light green (magnification 3000X) and orange (magnification 600X) regions, respectively. Whereas panels (C, D) show SEM-EDS spectra acquired from light green and orange regions, respectively. 


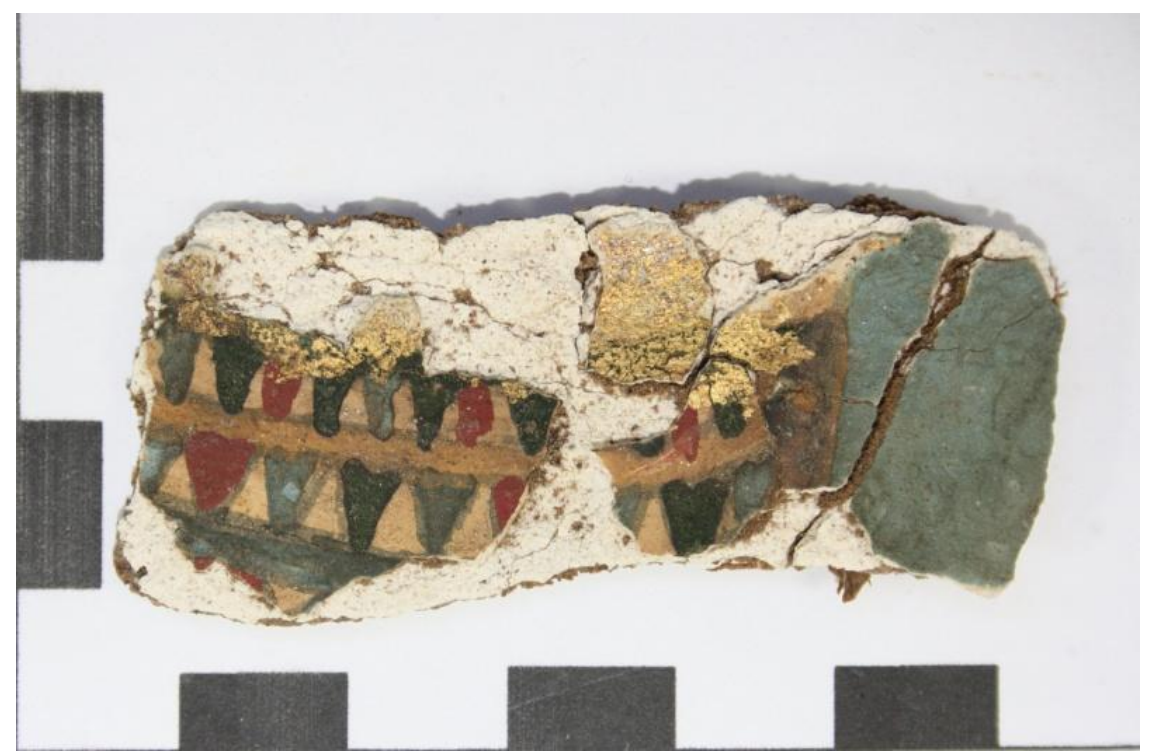

Figure 1. A small fragment of the cartonnage

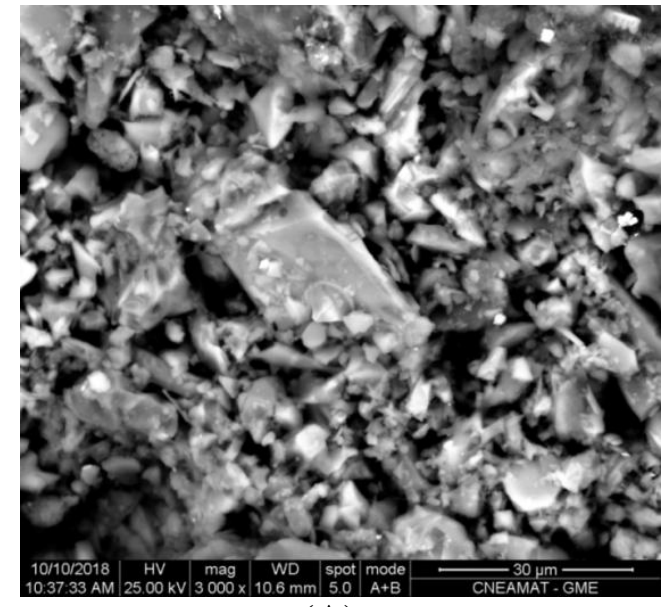

(A)

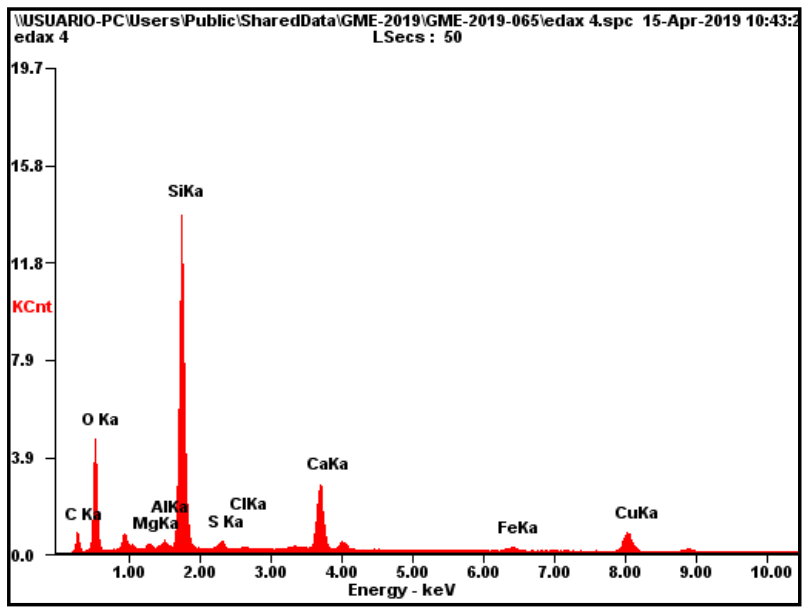

(C)

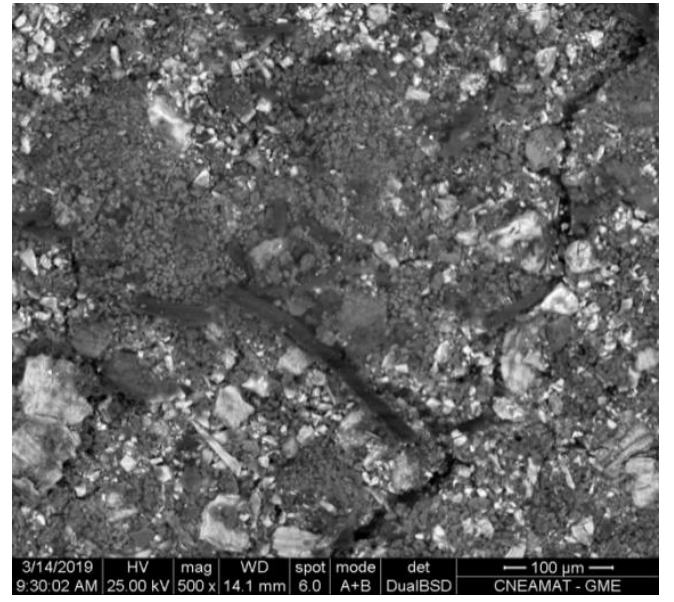

(B)

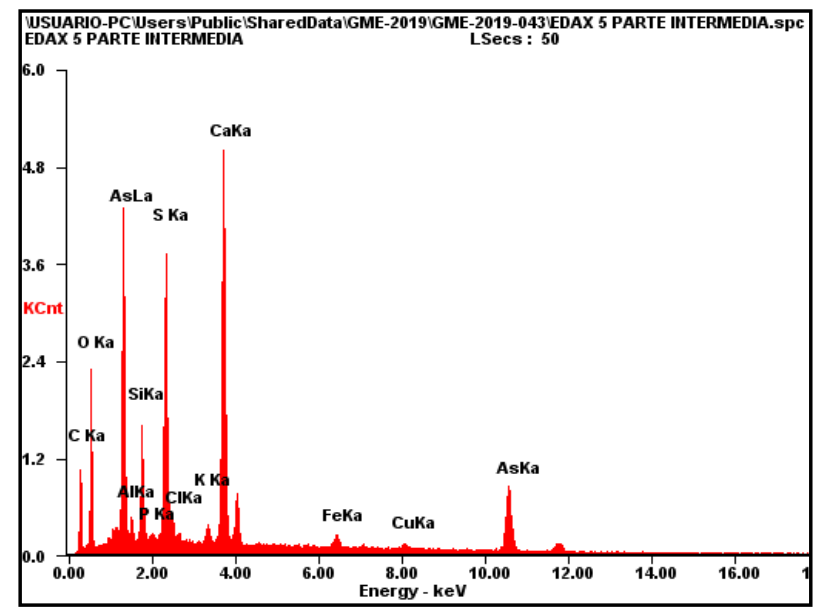

(D)

Figure 2. Backscattering BS images obtained from the light green (A) and orange (B) regions. EDS spectra from the light green $(C)$ and orange (D) pigments. 